\section{Intrinsically Contaminated Alcohol-Free Mouthwash Implicated in a Nosocomial Outbreak of Burkholderia cepacia Colonization and Infection}

TO THE EDITOR-Burkholderia cepacia has been linked to nosocomial outbreaks caused by contamination of medical devices, ${ }^{1}$ parenteral and nebulized medication, ${ }^{2-4}$ antiseptic solutions, and other environmental sources. ${ }^{5,6}$ It has also been implicated in 2 nosocomial outbreaks caused by contamination of alcohol-free mouthwash. ${ }^{7,8}$ Nevertheless, sterilization of mouthwash solutions is not mandatory in Spain, and these are widely used in patients undergoing mechanical ventilation. Such patients are highly vulnerable to pathogens that typically cause upper respiratory tract infection because of their inability to maintain the mucociliary and cough mechanisms that normally protect the lower respiratory tract. ${ }^{9}$

Between July 2004 and July 2005 at our teaching hospital in Las Palmas (Canary Islands, Spain), 37 adult patients had cultures positive for $B$. cepacia. Of the 37 isolates, 35 were from the respiratory tracts of patients in the intensive care unit (ICU), and 2 isolates were from patients in other areas of the hospital who had not been in the ICU during the study period. This letter summarizes our experience with the management of this large outbreak.

A case of infection or colonization was defined as detection of $B$. cepacia in culture of a specimen from any body site of any patient at the hospital from July 31, 2004 through July 22, 2005 (the epidemic period). Microbiological records were reviewed to identify all isolates of $B$. cepacia during the preepidemic period (January 1, 2000 through July 30, 2004) and the epidemic period. As part of the epidemiological investigation, we collected environmental samples and reviewed work schedules of healthcare workers, mainly with regard to respiratory therapy techniques used. As part of the intervention, contact isolation precautions were initiated for all patients identified as having had a culture positive for $B$. cepacia. Centers for Disease Control and Prevention definitions for nosocomial infections were not used.

A total of 37 patients had cultures positive for $B$. cepacia and met the case definition; only 4 patients ( 1 of which was an ICU patient) were identified during the pre-epidemic period. Case patients ranged in age from 16 to 77 years (mean, 49.9 years), and $57 \%$ were male. They had been admitted to the hospital with various diagnoses. None had medical conditions associated with $B$. cepacia infection (eg, cystic fibrosis or chronic granulomatous disease). Eleven patients died, but attributable mortality was not calculated. The rates of $B$. $c e-$ pacia colonization or infection in the pre-epidemic and ep- idemic periods were also not compared because of the lack of such information on the clinical records.

All ICU patients had undergone intubation and mechanical ventilation during their ICU stay. As a part of routine oral care for mechanically ventilated patients, they had had their mouths swabbed with an alcohol-free mouthwash; the active ingredient in this product is hexetidine $0.1 \%$. The 2 non-ICU patients received mouthwash as a part of routine care for enteral feeding.

As part of our epidemiological study, a Medline search was made with the terms "B. cepacia" and "nosocomial outbreak" on July 13, 2004, which identified 41 references; the studies of Bernstein et al. ${ }^{7}$ and Matrician et al. ${ }^{8}$ were crucial in performance of our environmental investigation. Considering the epidemiology of the microorganism and the previous findings, ${ }^{1-8}$ we decided to collect moist samples first, including antiseptics, tap water, and mouthwash solution.

Samples were cultured in blood, chocolate, and McConkey agar. The identification was made with the API 20 NE system (BioMérieux) and the 6W-Wider (Soria Melguizo). All the isolates showed the same antimicrobial susceptibility pattern. Cultures of samples from unopened $15-\mathrm{mL}$ bottles of the mouthwash yielded B. cepacia (more than 1,000 colony-forming units per $\mathrm{mL}$ ). Cultures of samples from tap surfaces yielded isolates of the Pseudomonas fluorescens-putida group. No other environmental samples revealed contamination.

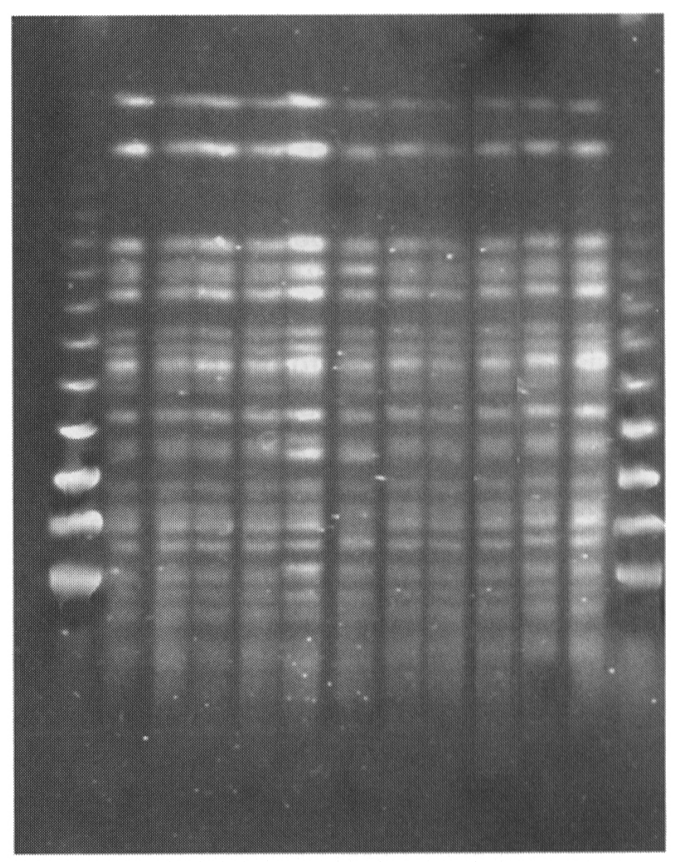

FIGURE. Pulsed-field gel electrophoresis patterns for isolates of Burkholderia cepacia recovered from 5 patients and 6 samples of mouthwash involved in the outbreak. 
Other potential reservoirs (eg, shaving cream, povidoneiodine solution, water supplies, and an alcohol-based mouthwash) were culture negative for $B$. cepacia.

Samples were obtained from unopened bottles of mouthwash distributed around the hospital that belonged to different batches, and the samples were cultured. Batches distributed from August 2004 through September 2004 were not contaminated. Batches distributed from April-2005 through July 2005 were highly contaminated. No other samples were available for analysis. The distribution of contaminated batches matched the substantial increase in the incidence of $B$. cepacia infection or colonization.

Strains from 5 patients and 6 mouthwash samples were submitted for identification to our reference center (National Center of Microbiology, Health Institute Carlos III, Majadahonda, Madrid, Spain). Pulsed-field gel electrophoresis of $X b a I$-digested genomic DNA was performed with a Chief DRIII system (Bio-Rad) according to conditions described elsewhere ${ }^{10}$ with several modifications. Briefly, electrophoresis was carried out in a $1.2 \%$ agarose gel for 22 hours at $6 \mathrm{~V} / \mathrm{cm}$ with pulse times ranging from 5 to 35 seconds. Molecular mass markers were concatameters of phage $\lambda$ New England (Biolabs, UK). Electrophoretic patterns showed that all the strains were identical (Figure).

On July 18, the use of the mouthwash product in our hospital was discontinued, and the last isolate of $B$. cepacia was obtained on July 22, 2005. The methods of production and distribution and the extent of use of this mouthwash in other hospitals are now being investigated by the Spanish Department of Health. At the moment, no information is available on the rate of $B$. cepacia infection or colonization at other hospitals that used the same product distributed by the same company as at our hospital.

Our findings strongly suggest that intrinsically contaminated alcohol-free mouthwash solution was the source of this large outbreak involving predisposed adults in ICU. To date, 2 similar outbreaks in which B. cepacia was isolated from culture of respiratory tract specimens from patients without cystic fibrosis have been traced to intrinsically contaminated alcohol-free mouthwash in North America. ${ }^{7,8}$

More-thorough surveillance of microbiological contamination of alcohol-free products used in adults predisposed to infection should be mandatory. These findings highlight the importance of hospital surveillance and investigation of unusual clusters of infection and colonization to promptly identify unexpected sources of pathogens and to protect patients at risk.

Jesús Molina-Cabrillana, $\mathrm{PhD}$; Margarita Bolaños-Rivero, $\mathrm{MD}$; Eva E. Alvarez-León, $\mathrm{PhD}$; Antonio M. Martín Sánchez, $\mathrm{PhD}$; Manuel Sánchez-Palacios, PhD; David Alvarez, BSc; Juan A. Sáez-Nieto, PhD
From the Medicine Preventive Service (J.M.-C., E.E.A.-L.), the Microbiology Service (M.B.R., A.M.M.-S.), and the Intensive Care Unit (M.S.-P.), Complejo Hospitalario Materno-Insular, Las Palmas, Gran Canaria, Canary Islands, and the Bacteriology Service, National Center of Microbiology, Health Institute Carlos III, Majadahonda, Madrid (D.A., J.A.S.-N.), Spain.

Address reprint requests to: Jesús Molina Cabrillana, Avda. Marítima del Sur s/n, 35016 Las Palmas de Gran Canaria, Spain (jmolcab@gobiernodecanarias .org)

Infect Control Hosp Epidemiol 2006; 27:1281-1282

(C) 2006 by The Society for Healthcare Epidemiology of America. All rights reserved. 0899-823X/2006/2711-0028\$15.00.

\section{REFERENCES}

1. Berthelot P, Grattard F, Mallaval FO, Ros A, Lucht F, Pozzetto B. Epidemiology of nosocomial infections due to Pseudomonas aeruginosa, Burkholderia cepacia and Stenotrophomonas maltophilia. Pathol Biol 2005; 53:341-348.

2. Balkhy HH, Cunningham G, Francis C, et al. A National Guard outbreak of Burkholderia cepacia infection and colonization secondary to intrinsic contamination of albuterol nebulization solution. Am J Infect Control 2005; 33:182-188.

3. Pegues CF, Pegues DA, Ford DS, et al. Burkholderia cepacia respiratory tract acquisition: epidemiology and molecular characterization of a large nosocomial outbreak. Epidemiol Infect 1996; 116:309-317.

4. Reboli AC, Koshinski R, Arias K, Marks-Austin K, Stieritz D, Stull TL. An outbreak of Burkholderia cepacia lower respiratory tract infection associated with contaminated albuterol nebulization solution. Infect Control Hosp Epidemiol 1996; 17:741-743.

5. Oie S, Kamiya A. Microbial contamination of antiseptics and disinfectants. Am J Infect Control 1996; 24:389-395.

6. Moreira BM, Leobons MB, Pellegrino FL, et al. Ralstonia pickettii and Burkholderia cepacia complex bloodstream infections related to infusion of contaminated water for injection. $J$ Hosp Infect 2005; 60:51-55.

7. Bernstein B, Dineen T, Kehl S, Wilson P, Sohnle P. Outbreak of Burkholderia cepacia colonization and infection related to contaminated oral mouthwash. In: Program and abstracts of the 34th Annual Meeting of the Infectious Diseases Society of America; September 1996; New Orleans, LA. Abstract.

8. Matrician L, Ange G, Burns S, et al. Outbreak of nosocomial Burkholderia cepacia infection and colonization associated with intrinsically contaminated mouthwash. Infect Control Hosp Epidemiol 2000; 21:739-741.

9. Beck-Sague CM, Sinkowitz RL, Chinn RY, Vargo J, Kaler W, Jarvis WR. Risk factors for ventilator-associated pneumonia in surgical intensive care unit patients. Infect Control Hosp Epidemiol 1996; 17:374-376.

10. Kanellopou M, Pournaras S, Iglezos, H, Sharmoutsou N, Papagrangas E, Maniatis AN. Persistent colonization of nine cystic fibrosis patients with an Achromobacter (Alcaligenes) xylosoxidans clone. Eur J Clin Microbiol Infect Dis 2004; $23: 336-339$.

\section{Analysis of Costs Attributable to an Outbreak of Severe Acute Respiratory Syndrome at a French Hospital}

TO THE EDITOR-Severe acute respiratory syndrome (SARS), which is caused by the SARS-associated coronavirus 